\title{
Case Report: Recurrence of Scrotal Lipoma
}

\section{Sushila B Ladumor $^{1 *}$ and Aalaa Salaheldin Kambal ${ }^{2}$}

${ }^{1}$ Consultant Radiologist, Clinical Imaging Department, Hamad Medical Corporation, HGH, Doha, Qatar

${ }^{2}$ Associate Consultant in Clinical Imaging Department, Body Imaging, Hamad Medical Corporation, Hamad General Hospital, Qatar

"Corresponding author: Ladumor SB, Consultant Radiologist, Clinical Imaging Department, Hamad Medical Corporation, Assistant Professor in Clinical Radiology, Weil Cornel Medical College, Qatar, Tel: +974-4492-8000; E-mail: drsbladumor@yahoo.com

Received: March 09, 2019; Accepted: May 12, 2019; Published: May 19, 2019

\begin{abstract}
The para-testicular mass lesions are usually of benign etiology in the majority of cases [1]. Lipoma is the most common extra testicular neoplasm and originates from the fat cells of the spermatic cord or subcutaneous fat in the scrotal wall. Whenever patient present with a scrotal mass, detailed history and local examination of inguinoscrotal regions are must. Malignant scrotal wall, Para testicular, or spermatic cord tumors are rare. After initial evaluation first Imaging study is Scrotal ultrasound and US is best modality that evaluate and confirm the exact location and organ of origin of a mass or rule out the presence of an inguinal hernia. It is very accurate in differentiating between an intra-testicular and extra testicular location and the size, shape, and echogenicity of the mass that will further helpful to narrow the differential diagnosis. Intra testicular masses need a formal workup, with serum tumor markers, according to the Imaging findings warrants immediate referral/ consultation with a urologist for further staging and intervention.
\end{abstract}

Keywords: Lipoma; Scrotal sac; Testicular; Extra testicular; Para testicular

Abbreviation: US (Ultrasound), MRI (Magnetic Resonance Imaging), O/E: On Examination, H/O (History of), FNAC (fine needle aspiration cytology)

\section{Introduction}

Extra testicular neoplasms are rare but clinically significant lesions that affect patients of all ages [2]. Scrotal swelling may be due to extra testicular and intra testicular lesions. Extra testicular scrotal masses (non-testicle and non-epididymis) are mostly mesenchymal in origin. We present a case of intra scrotal extra testicular lipoma. The patient was a 63-year-old gentle man with the chief complaint of painless swelling in the scrotum which had been noticed before about 3 years.

Citation: Ladumor SB, Kambal AS, et al. Case Report: Recurrence of Scrotal Lipoma. Clin Case Rep Open Access. 2019;2(2):126. 
www.yumedtext.com | May-2019

\section{Clinical Presentation}

63 year old male patient presented with recurrent right hemi scrotal swelling? Lipoma. Currently patient noticed slight increase in size.

Previous history from Cerner: Patient had H/O previous similar history and had US and MRI in 2015 and diagnosed as lipoma. H/O right testicular mass excision 2 years ago -HGH - 2015, Histopathology: Fragments of traumatized and inflamed lipoma $\mathrm{O} / \mathrm{E}$ - left testis and epididymis are normal enlarged right testis. Not tender palpable lesion at lower pole of Right testis.

\subsection{Imaging work-up}

US requested in 2019: US revealed well-defined oblong focal lesion measuring about $8.8 \times 4.9 \times 3.1 \mathrm{~cm}$ is noted adjacent to the right testis. It appears heterogeneous iso to hyperechoic with no internal vascularity, likely a lipoma. Right testis appears mildly compressed and displaced inferiorly. Patient had no history of trauma so diagnosis of lipoma (recurrence in view of previous surgery) was considered.

MRI in 2019: Multi planner, multi sequential MRI of scrotum reveal re-demonstration of right extra testicular mass showing signal intensity similar to subcutaneous fat which shows suppression in fat sat sequences indicating presence of fat. Also small area of signal drop in opposed phase compared to in-phase representing intra cellular fat. Minimal septal enhancement was present. Findings are keeping with recurrence of lipoma with possible superadded infection/inflammation. (In view of history of previous removal of lipoma). Previous Imaging: One image of MRI in 2015 (Figure 5 image \#f)

\section{Discussion}

Mesenchymal tumors of the scrotum are very rare. These include lipomas, liposarcomas, ficrosarcomas, fibromas, fibrolipomas and myxochondrasarcomas.

Lipomas are the most common extra testicular neoplasm of the scrotum, occurring in approximately $22 \%$ of patients who undergo hernia repair [1] and from Para testicular tissues and spermatic cord, of about $45 \%$ of Para testicular masses. This lesion is mostly discovered incidentally as non-tender scrotal mass and seen in patients over a wide age range [2]. In rare cases, a lipoma can originate outside the spermatic cord or in the subcutaneous fat [3].

Ultrasonography, CT and MRI scans play key role in the detection and evaluation of scrotal lesions. US is easily available, cheap and non-ionizing imaging modality, forms the first line of investigation. With high resolution probe of about 10-12 MHZ easily detect weather scrotal lesion is intra testicular or extra testicular and also detect the nature of lesion as cystic or solid. Also evaluate skin involvement or skin lesion as well. Solid extra testicular masses, like we reported, are more difficult to diagnose. While most such tumors are benign, uncertainty might arise sometimes especially in huge masses. Sometimes FNAC cannot clearly distinguish a benign lesion from a malignant one due to heterogeneity of the mass. Routine radiological imaging (US) can also confuse between a well circumscribed liposarcoma and lipoma. MR imaging is very helpful in confusing cases as it is the more sensitive in differentiating between benign mass from a malignant and helpful for plan of 
management. A discrete homogenous encapsulated fatty mass is most certainly a lipoma. However a large lipoma may have few non adipose components as well like muscle fibers, blood vessels and fibrous tissues. These findings mimic those of a well differentiated liposarcoma. However absence of diffusion restriction within mass in absence of hemorrhage and no enhancement can be very much helpful in differentiating benign versus malignant nature of mass [2-4]. At sonography, lipomas are typically well-defined and uniformly hyperechoic, although this finding is variable (FIG. 1). Internal flow is often not seen at color Doppler imaging. At computed tomography (CT), lipomas demonstrate homogeneous attenuation similar to that of subcutaneous fat [5-7]. Magnetic resonance (MR) imaging can be useful for confirming the diagnosis of a lipoma. Lipomas most often have high signal intensity on T1- and T2-weighted images shows drop of signal in fat sat sequences and no post-contrast enhancement (FIG. 2-5).

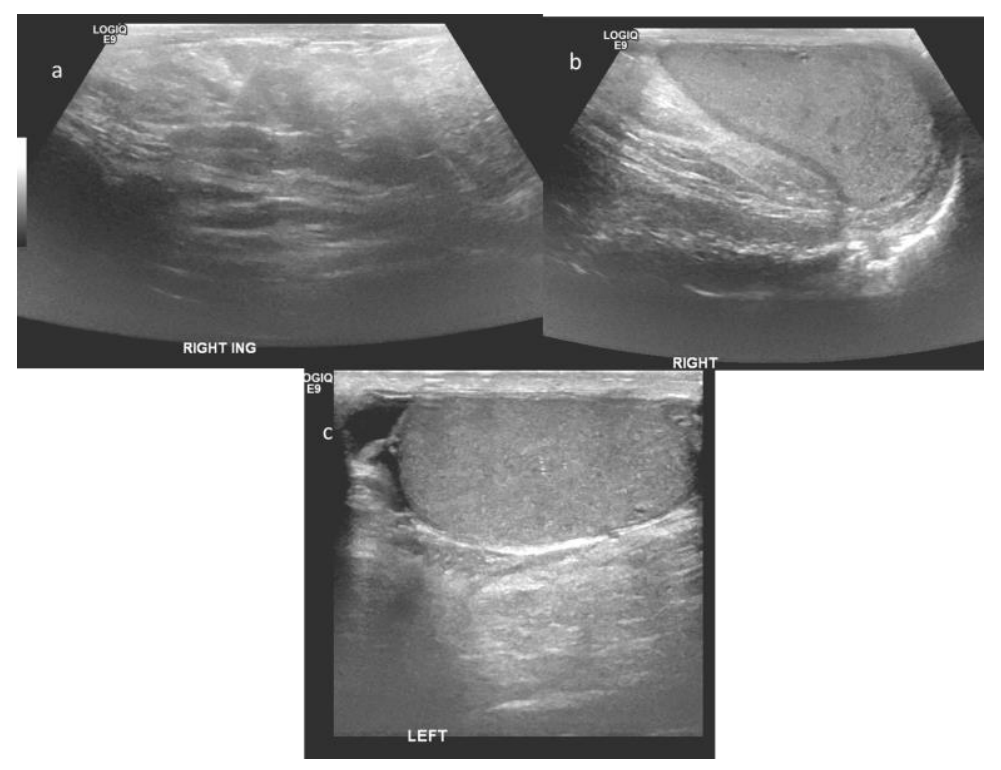

FIG. 1. A well-defined oblong focal lesion measuring about $8.8 \times 4.9 \times 3.1 \mathrm{~cm}$ is noted adjacent to the right testis. It appears heterogeneous iso to hyperechoic with no internal vascularity on Doppler (Image not seen), likely a lipoma.

Right testis appears mildly compressed and displaced inferiorly.

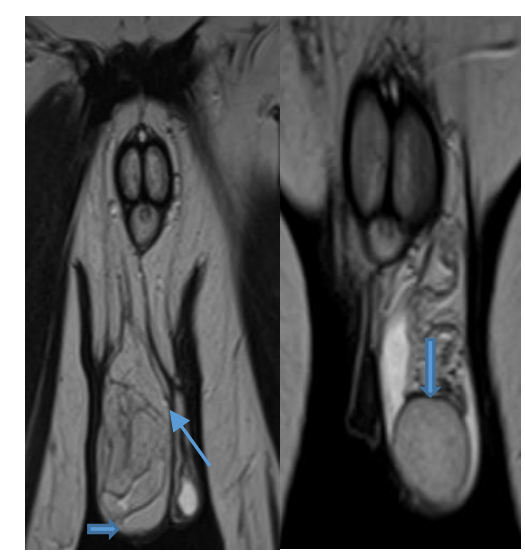

FIG. 2. T2 TSE coronal Image (a \& b): Large heterogeneous predominantly hyper intense lesion (similar to subcutaneous fat) in right scrotal sac (thin arrow in image \# a) with right testis is displaced and mildly compressed (thick transverse arrow in Image \#a), otherwise appears unremarkable and similar T2 signal with normal left testis. (Vertical arrow in Image \# b). 


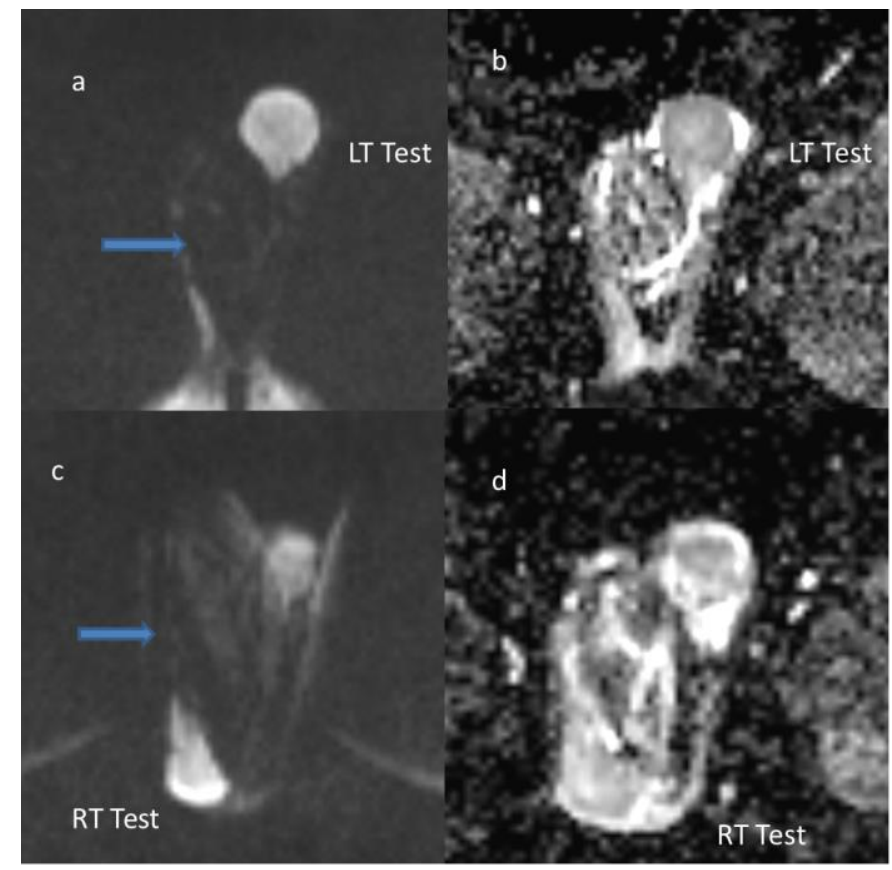

FIG. 3. Axial Section Diffusion (a \& c) with corresponding ADC (b \& d): Showing mildly compressed and displaced right testis, otherwise both testis appears normal with no focal testicular lesion. No area of diffusion restriction in right scrotal lesion (Transverse arrow in all Image).

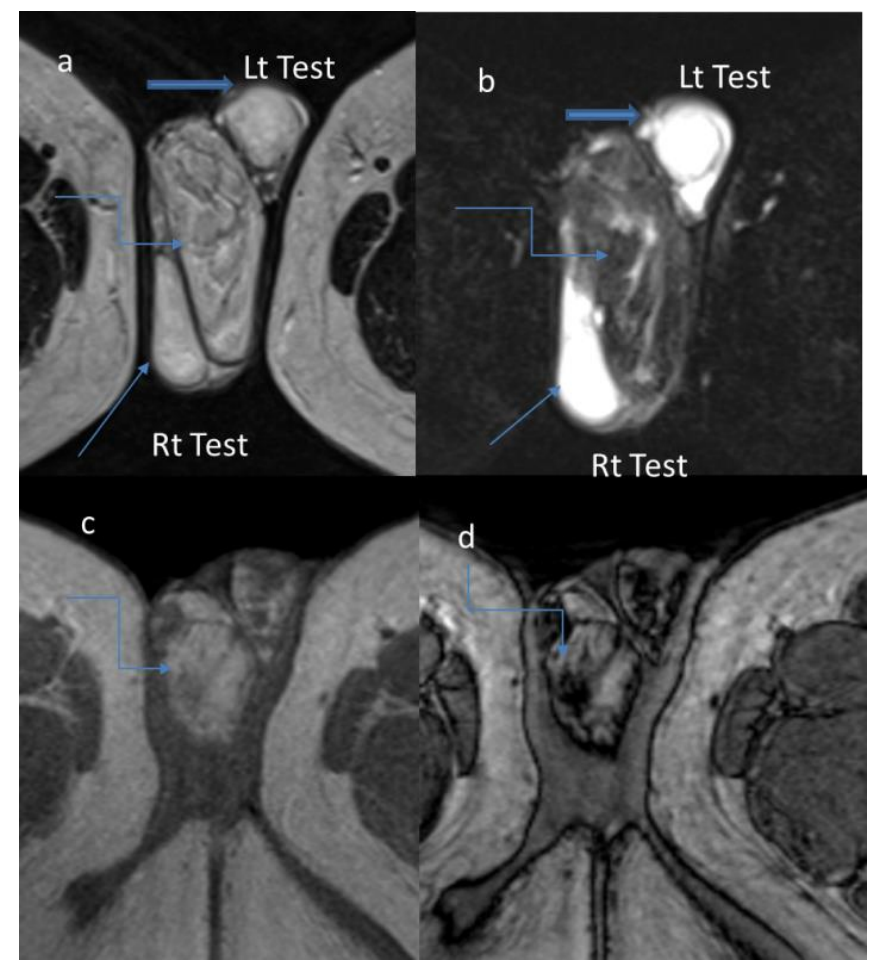

FIG. 4. Axial section: Image $a=\mathbf{T} 2$ tse, $b=\mathbf{T} 2$ fat sat, $c=I n-p h a s e$ and $\mathbf{d}=$ opposed phase $=$ Re-demonstration of large right scrotal sac mass 9 curved arrow in image a, b, c \& d) showing near similar signal as subcutaneous fat which shows suppression in fat sat similar to suppression of subcutaneous fat indicating fatty component which also shows small area of signal drop in opposed phase represents intra cellular fatty component. 


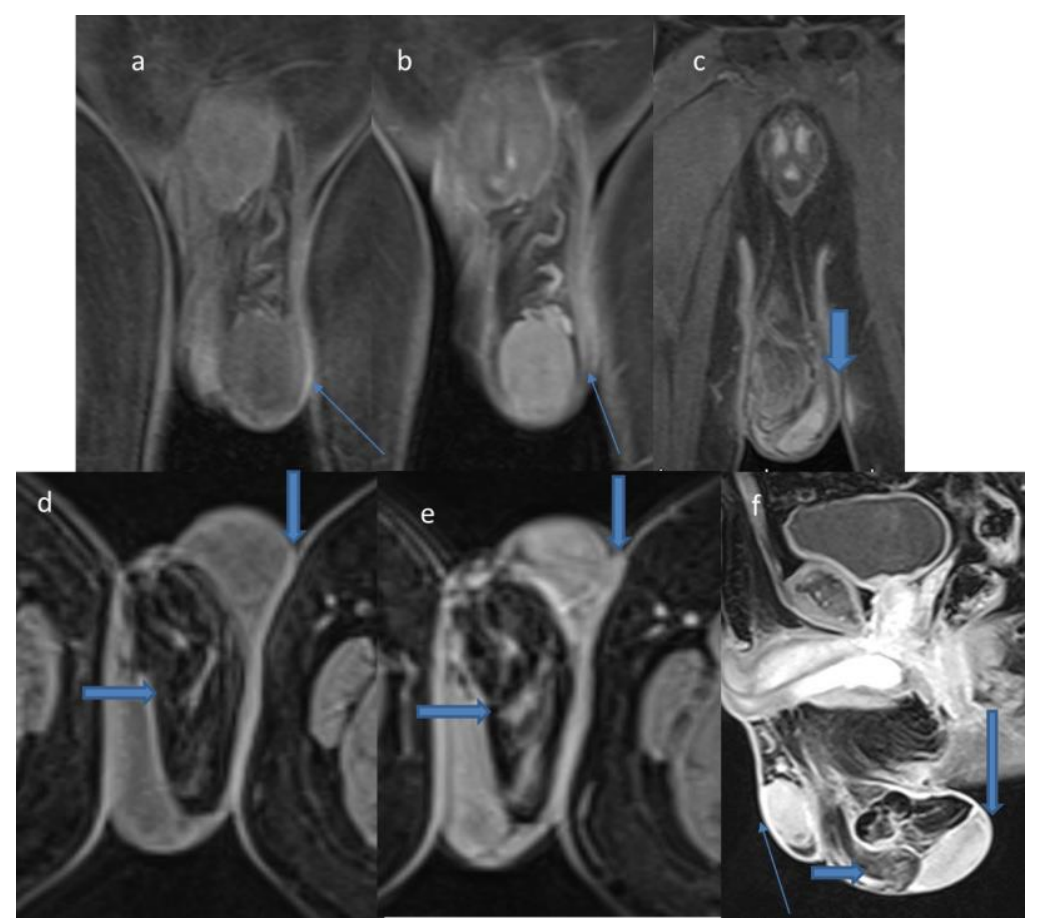

FIG. 5. Pre contrast Coronal (a) and Axial (d), Post-contrast coronal (b \& C) Axial (f). Previous post-contrast sagittal Image of 2015. Right scrotal mass shows fat suppression (transverse arrow in image d, e \& f) with minimal internal septal enhancement which is same in size and pattern before three years. Normal enhancing both testis (Right testis shown by vertical arrow in image \# c, d, e \& f, Left testis shown by thin arrow in image \# a, b \& f).

\section{Classification of Scrotal Tumors}

- Benign lesions

1. Lipoma (most common)

2. Leiomyoma of the scrotum

3. Neurofibroma

4. Granular cell tumor

5. Angiomyofibroblastoma-like tumor

6. Fibrous pseudotumor

7. Fibrous hamartoma of infancy

- Malignant lesions

Malignant lesions of the scrotum are rare.

1. Metastasis

2. Liposarcoma

3. Leiomyosarcoma of the scrotum

4. Malignant fibrous histiocytoma (MFH)

5. Rhabdomyosarcoma

6. Primary dermal lesions extending into the deeper layers of the scrotum (e.g. melanoma) 
www.yumedtext.com | May-2019

\section{Conclusion}

US is a useful imaging technique for evaluating patients with a painless scrotal swelling. Extra testicular scrotal masses are rare tumors which can be large and may cause significant diagnostic challenge. Clinical history with local examination along with radiological imaging especially USG and MR plays major role in reaching to diagnosis which is very helpful for management plan. Surgery remains the treatment of choice according to diagnosis. Many of lesions are benign, however sarcomas can occur and it can be suspected in cases of large heterogeneous lesions masses and invade or infiltrate other adjacent structures $[3,4]$.

\section{REFERENCES}

1. Wolfman DJ, Marko J, Gould CF, et al. Mesenchymal Extratesticular Tumors and Tumorlike Conditions: From the Radiologic Pathology Archives. Radiographics. 2015;35(7):1943-54.

2. SA Akbar, Sayyed TA, Jafri SZH, et al. Multimodality Imaging of Paratesticular Neoplasms and Their Rare Mimics. Radiographics. 2003;23(6):1461-76.

3. Srivastava KN, Agarwal A, Vikram SSS, et al. Huge scrotal lipoma posing a diagnostic dilemma: A case report and review of literature. Urol Case Rep. 2017;15:39-41.

4. Cassidy FH, Ishioka KM, McMahon CJ, et al. MR Imaging of Scrotal Tumors and Pseudotumors. Radiographics. 2010;30(3):665-83.

5. Fabiani A, Principi E, Filosa A, et al. An unusual case of primary intrascrotal lipoma. Arch Ital Urol Androl. 2016;88(4):345-6.

6. Rafailidis V, Robbie H, Konstantatou E, et al. Sonographic imaging of extra-testicular focal lesions: comparison of grey-scale, colour Doppler and contrast-enhanced ultrasound. Ultrasound. 2016;24(1):23-33.

7. Woodward PJ, Schwab CM, Sesterhenn IA. Extratesticular Scrotal Masses: Radiologic-Pathologic Correlation. Radiographics. 2003;23(1):215-40. 